\title{
Fenilquetonuria de diagnóstico neonatal y lactancia materna
}

\author{
Verónica Cornejo $\mathrm{E}^{\mathrm{a}}$, Viviana Manríquez $\mathrm{E}^{\mathrm{a}}$, \\ Marta Colombo C, Paulina Mabe S, Mónica Jiménez $M$ a, \\ Alicia De la Parra $\mathbf{C}^{\mathbf{b}}$, Alf Valiente $\mathbf{G}^{\mathrm{c}}$, Erna Raimann B. \\ Phenylketonuria diagnosed during \\ the neonatal period and breast \\ feeding
}

Background: Phenylketonuria (PKU) is due to of a defect in the phenylalanine hydroxylase gene (12q22-24.1) leading to hyperphenylalaninemia. Treatment consists in a low phenylalanine (Phe) diet. Aim: To evaluate the evolution of early diagnosed PKU children, receiving direct breast feeding, and a special formula without Phe, during their first six months of life. Patients and methods: Nineteen PKU children diagnosed in the neonatal period (19.29 \pm 13.8 days of age), treated with breast feeding and formula without Phe since diagnosis, were studied. Intake of calories, proteins and dietary Phe were quantified. Blood Phe, nutritional status and psychomotor development were also measured. Results: The diet that these children received during the 6 months period of study, had a mean of $127 \pm 19.9 \mathrm{Kcal} / \mathrm{kg} /$ day, $1.95 \pm 0.3 \mathrm{~g}$ protein/kg/day and $35.3 \pm 9.5 \mathrm{mg}$ Phe/ $\mathrm{kg} /$ day. Fifteen children maintained the blood level of Phe under $8 \mathrm{mg} / \mathrm{dl}$, considered an excellent metabolic control. Only 4 cases had intermittently high levels, between 10-12 mg/dl. At 6 months of age, $74 \%$ of the children maintained breast feeding as the only source of Phe. Sixty three percent had a normal nutritional status, $5.2 \%$ were at nutritional risk and $31.6 \%$ were overweight. Eighty one percent had a normal mental development. Conclusions: The use of direct breast feeding allows a good metabolic control and improves growth and development of early diagnosed PKU children (Rev Méd Chile 2003; 131: 1280-87).

(Key Words: Breast feeding; Phenylalanine hydroxylase; Phenylketonuria)

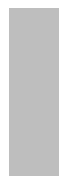

Recibido el 27 de marzo, 2003. Aceptado en versión corregida el 19 de agosto, 2003.
INTA, Universidad de Chile, Unidad de Nutrición Clínica.
a Nutricionista
b Psicóloga
c Bioquímico

Correspondencia a: MSc Verónica Cornejo E. Macul 5540,

Macul. Fax: 56-2-2941254. Fono: 56-2-6781491.

E mail: vcornejo@inta.cl 
L a fenilquetonuria clásica (Mc Kusick 261 600) (PKU) se produce por el defecto en el gen que codifica la enzima fenilalanina hidroxilasa (FAH), ubicado en el cromosoma 12q22-24.1. Se caracteriza por presentar nivel de fenilalanina (FA) en sangre sobre $20 \mathrm{mg} / \mathrm{dl}$. Si esta enfermedad no es diagnosticada y tratada precozmente, ocasiona deterioro progresivo en el sistema nervioso central, produciendo retardo mental moderado o profundo ${ }^{1}$. La incidencia para la PKU clásica es en promedio 1:10.000 recién nacidos $(\mathrm{RN})$ vivos $^{2}$. En Chile a 10 años de desarrollo del Programa Nacional de Pesquisa Neonatal de Fenilquetonuria se ha establecido una incidencia de 1:14.000 $\mathrm{RN}^{2,4,5}$.

Se ha demostrado ampliamente que el inicio del tratamiento en el período neonatal y la mantención del nivel de FA en suero menor a 10 $\mathrm{mg} / \mathrm{dl}(600 \mu \mathrm{mol} / \mathrm{l})$, previene el daño neurológico. El tratamiento es nutricional y consiste en restringir la ingesta de FA, lo que significa suspender de la dieta alimentos naturales ricos en proteínas de alto valor biológico, siendo necesario entregar una fórmula láctea especial sin FA o una mezcla de aminoácidos, que aporte las necesidades requeridas de proteínas y energía 3 .

Tradicionalmente, la dieta de la PKU suspendía la lactancia materna e introducía el sustituto lácteo bajo o sin FA en combinación con fórmula láctea de inicio convencional y mantenía el nivel en sangre de este aminoácido entre 2 y $6 \mathrm{mg} / \mathrm{dl}$ y permitía el normal crecimiento y desarrollo ${ }^{8}$.

Estudios recientes han demostrado que el uso de leche materna es una herramienta asequible para el tratamiento en PKU, porque contiene menor cantidad de FA que la leche de vaca ( $47 \mathrm{mg}$ y $195 \mathrm{mg} \mathrm{FA} / 100 \mathrm{ml}$, respectivamente), aporta nucleótidos mejorando la biodisponibilidad proteica y logra mantener el nivel en sangre de FA entre 2 y $6 \mathrm{mg} / \mathrm{dl}$, considerado un excelente balance metabólico ${ }^{9,11}$.

Riva y cols, evaluaron el efecto a corto plazo de la lactancia materna sobre el desarrollo cognitivo en niños $\mathrm{PKU}^{10}$. A los 9,5 años de edad midieron el coeficiente intelectual (CI) en niños PKU que habían recibido lactancia materna exclusiva durante los primeros 10 a 20 días de vida (previo al diagnóstico) y en niños PKU que habían recibido fórmulas de inicio para lactantes. Al comparar ambos grupos, se detectaron diferencias significativas entre los CI, siendo mejor en los niños PKU que habían recibido precozmente lactancia materna ${ }^{10}$.

En 1998 el programa de seguimiento de PKU del Instituto de Nutrición y Tecnología de los Alimentos (INTA), Universidad de Chile, implementó el uso de la lactancia materna en combinación con una fórmula especial sin FA. Todos los niños PKU ingresan a este programa donde son evaluados por un equipo conformado por pediatra, neurólogo, nutricionista, psicólogo, bioquímico y asistente social ${ }^{6}$.

El objetivo de este estudio fue evaluar el seguimiento durante los primeros 6 meses de edad, en niños PKU diagnosticados en el período neonatal, que recibieron tratamiento nutricional desde el diagnóstico, utilizando lactancia materna y fórmula especial sin FA.

\section{PACIENTES Y MÉTODO}

Este es un estudio retrospectivo de corte transversal. La muestra estuvo conformada por 19 niños con PKU (9 hombres y 10 mujeres) que ingresaron al programa de seguimiento entre abril de 1998 y febrero de 2000. La edad de diagnóstico promedio fue de 19,9 \pm 13 , 8 días y el nivel de FA sérica inicial fue $21,14+8,53 \mathrm{mg} / \mathrm{dl}$.

Todos recibieron tratamiento nutricional desde el momento del diagnóstico. La dieta consistió en restringir la ingesta de FA y proporcionar los requerimientos nutricionales de proteínas, calorías y FA según peso, edad y nivel de FA en sangre ${ }^{12}$. Los requerimientos de nutrientes fueron proporcionados a través de una fórmula especial baja 0 sin FA y leche materna directa del pecho.

Protocolo de tratamiento de la PKU con lactancia materna. Se estimó el volumen total de líquido a ingerir, según la recomendación establecida por la Recommended Dietary Allowance (RDA) para niños menores de 6 meses de edad ( $150 \mathrm{ml} / \mathrm{kg} /$ día), en relación al peso real del niño ${ }^{13}$.

En la primera semana de tratamiento, se suspendió la lactancia materna y se entregó $100 \%$ del líquido requerido como fórmula especial sin FA. Se completó el aporte de calorías con maltosa dextrina y aceite vegetal, de preferencia de soja, de acuerdo a las recomendaciones establecidas por la RDA para edad y peso real ${ }^{12}$. Semanalmen- 
te se midió el nivel de FA en sangre con el método de inhibición bacteriana de Guthrie y según este nivel se realizó el ajuste de ingesta de $\mathrm{FA}^{12}$. $\mathrm{Al}^{\circ}$ día de tratamiento se evaluó el nivel de FA en sangre. Si éste había descendido bajo los 8-10 mg/ dl, se disminuyó la fórmula especial en $50 \%$ y se completó el volumen con leche materna, colocando el niño al pecho después de cada mamadera. Esta operación se realizaba cada $3 \mathrm{~h}$ y la madre debía registrar cada alimentación considerando el volumen de fórmula especial, el tiempo de amamantamiento y la frecuencia horaria.

Protocolo de evaluación del nivel de FA en sangre. $\mathrm{Si}$ el nivel de FA se encontraba entre $2-6 \mathrm{mg} / \mathrm{dl}$ se mantenía la prescripción. -Si estaba bajo $2 \mathrm{mg} / \mathrm{dl}$, se disminuía la fórmula especial en $25 \%$, aumentando indirectamente el volumen de leche materna y la cantidad de FA dietaria. -Si el nivel de FA en sangre estaba entre 6 y $10 \mathrm{mg} / \mathrm{dl}$, se aumentaba el volumen de la fórmula especial sin FA en $25 \%$, y se disminuía de forma indirecta la ingesta de leche materna. -Si el nivel de FA se encontraba sobre 10 $\mathrm{mg} / \mathrm{dl}$, se aumentaba la fórmula en $50 \%$ del total de líquidos prescritos, según el peso real del niño.

La ingesta de nutrientes y fenilalanina de los pacientes en estudio se obtuvo mediante la encuesta de recordatorio de $24 \mathrm{~h}$. Se calcularon promedios y desviaciones estándares de estos parámetros y los resultados obtenidos se compararon con las recomendaciones nutricionales establecidas por la RDA, para pacientes con PKU en tratamiento $^{12}$
La evaluación del estado nutricional de los niños PKU se realizó mensualmente durante los primeros 6 meses de edad y se examinó: Peso/ Edad (P/E), Talla/Edad (T/E), Peso/Talla (P/T). Se utilizó como estándar las tablas de la National Center for Health Statistics (NCHS) ${ }^{14}$ y los datos se expresaron en puntaje $\mathrm{z}$.

Se evaluó el desarrollo mental (MDI) y motor (PDI) a los 4 y 6 meses de edad, con la escala de desarrollo infantil de Bayley.

Para los análisis estadísticos de la información obtenida, se utilizaron los programas computacionales Excel y EPI INFO 6.0. Se calcularon promedios, desviación estándar, porcentajes y puntaje z.

\section{Resultados}

La muestra estuvo constituida por 19 lactantes (9 hombres y 10 mujeres) fenilquetonúricos. La ingesta por kilo de peso de: calorías, proteínas y FA a los 6 meses de tratamiento fue: $126 \pm 17,9 \mathrm{kcal}, 2,04 \pm 0,3$ gr y $37,6 \pm 8,6 \mathrm{mg}$ respectivamente (Tabla 1 ).

La ingesta diaria de proteínas fluctuó entre $7,9 \pm 1,3 \mathrm{~g} /$ día el primer mes de edad y $15,8 \pm 7,9 \mathrm{~g} /$ día el $6^{0}$ mes. Al separar según tipo de alimento, se comprobó que la fórmula especial sin FA

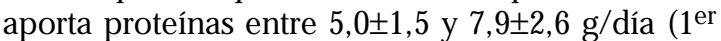
y 6 o mes de edad), lo que equivale al $50-60 \%$ del total de proteínas. La leche materna estimada aporta entre $2,9 \pm 0,7$ a 3,8 $\pm 3,1 \mathrm{~g} /$ día de nitrógeno no proteico, correspondiendo al 24\%-35\% del total (Tabla 2).

\section{Tabla 1. Ingesta de calorías, proténas y FA de 19 niños PKU de diagnóstico precoz en tratamiento y recomendaciones de RD A durante el primer semestre de vida}

\begin{tabular}{|c|c|c|c|c|c|c|}
\hline $\begin{array}{l}\text { Edad } \\
\text { (ms) }\end{array}$ & $\begin{array}{c}\text { Ingesta } \\
\text { Calorías } \\
\text { cal/kg }\end{array}$ & $\begin{array}{c}* \text { RDA } \\
\mathrm{cal} / \mathrm{kg}\end{array}$ & $\begin{array}{c}\text { Ingesta } \\
\text { Proteínas } \\
\text { gr/kg }\end{array}$ & $\begin{array}{c}\text { *RDA } \\
\text { Prot gr/kg }\end{array}$ & $\begin{array}{c}\text { Ingesta } \\
\mathrm{FA} \\
\mathrm{mg} / \mathrm{kg}\end{array}$ & $\begin{array}{c}\text { *RDA } \\
\text { FA } \mathrm{mg} / \mathrm{kg}\end{array}$ \\
\hline $1^{0}$ & $145,9 \pm 29,0$ & 120 & $2,13 \pm 0,4$ & 2,2 & $31,8 \pm 6,9$ & 45 \\
\hline $2^{\underline{o}}$ & $137,2 \pm 20,5$ & 115 & $2,14+0,4$ & 2,2 & $37,9 \pm 10$ & 45 \\
\hline 30 & $121,5 \pm 17,2$ & 105 & $1,84 \pm 0,3$ & 2,2 & $36,7 \pm 9,7$ & 45 \\
\hline $4 \underline{0}$ & $115,3 \pm 17,6$ & 100 & $1,75 \pm 0,3$ & 2,0 & $33,2 \pm 9,7$ & 32,5 \\
\hline $5^{0}$ & $119,1 \pm 17,3$ & 98 & $1,80 \pm 0,3$ & 1,8 & $34,5 \pm 12$ & 32,5 \\
\hline $6^{0}$ & $126,8 \pm 17,9$ & 98 & $2,04 \pm 0,3$ & 1,6 & $37,6 \pm 8,6$ & 32,5 \\
\hline
\end{tabular}

*RDA 1996. 
Tabla 2. D istribución de ingesta de proteínas diarias según tipo de alimentos en niños con fenilquetonuria durante 6 meses de seguimiento

\begin{tabular}{|lcccccc|}
\hline Tipo alimento & $\begin{array}{c}1^{\mathrm{er}} \text { mes } \\
\text { gr. prot/día }\end{array}$ & $\begin{array}{c}2^{2} \text { mes } \\
\text { gr. prot/día }\end{array}$ & $\begin{array}{c}3^{\mathrm{er}} \text { mes } \\
\text { gr. prot/día }\end{array}$ & $\begin{array}{c}4^{\mathbf{0}} \text { mes } \\
\text { gr. prot/día }\end{array}$ & $\begin{array}{c}5^{\mathbf{0}} \text { mes } \\
\text { gr. prot/día }\end{array}$ & $\begin{array}{c}6^{\mathbf{0}} \text { mes } \\
\text { gr. prot/día }\end{array}$ \\
\hline Leche materna estimada & $2,9 \pm 0,7$ & $4 \pm 1,7$ & $4,7 \pm 2,4$ & $4,7 \pm 2,6$ & $4,3 \pm 3,2$ & $3,8 \pm 3,1$ \\
Fórmula infantil & - & $0,4 \pm 1,2$ & $0,5 \pm 1,3$ & $0,5 \pm 1,2$ & $1,4 \pm 1,9$ & $1,6 \pm 2,3$ \\
Leche especial sin FA & $5,0 \pm 1,5$ & $6 \pm 2,6$ & $5,3 \pm 1,9$ & $6 \pm 2,4$ & $6,8 \pm 2,3$ & $7,9 \pm 2,6$ \\
Otros alimentos & - & - & - & $0,2 \pm 0,4$ & $0,4 \pm 0,4$ & $2,5 \pm 3,1$ \\
Ingesta total & $7,9 \pm 1,3$ & $10,5 \pm 2,4$ & $10,5 \pm 1,8$ & $11,5 \pm 2,2$ & $12,9 \pm 2,3$ & $15,8 \pm 2,4$ \\
Gr Prot/kg/día & 2,13 & 2,14 & 1,84 & 1,75 & 1,8 & 2,04 \\
\hline
\end{tabular}

Tabla 3. D istribución de la ingesta diaria de fenilalanina de niños PKU, según tipo de alimentos

\begin{tabular}{|lcccccc|}
\hline \multirow{2}{*}{ Tipo alimento } & $1^{\mathrm{er}}$ mes & $2^{\underline{0}}$ mes & $3^{\mathrm{er}}$ mes & $4^{0}$ mes & $5^{0}$ mes & 60 mes \\
\cline { 2 - 7 } & \multicolumn{5}{c|}{ Fenilalanina mg/día } \\
\hline Leche materna estimada & $112 \pm 27$ & $154 \pm 66$ & $184 \pm 92$ & $182,5 \pm 99$ & $163 \pm 126$ & $107 \pm 114$ \\
Fórmula Iáctea de inicio & - & $18 \pm 55$ & $23,4 \pm 59$ & $23,4 \pm 53$ & $60 \pm 84$ & $73 \pm 100$ \\
Leche especial & $6,7 \pm 12$ & $14 \pm 21$ & $6,7 \pm 12$ & $9,5 \pm 14$ & $13 \pm 19$ & $13,8 \pm 21$ \\
Alimentos sólidos & - & - & - & $4,0 \pm 7,0$ & $8,6 \pm 10$ & $59 \pm 43$ \\
Ingesta total FA & $119,7 \pm 25$ & $186,1 \pm 57$ & $214 \pm 63$ & $219,3 \pm 66$ & $244,8 \pm 82$ & $290,4 \pm 59$ \\
\hline
\end{tabular}

Debido a que el tiempo de succión es menor, por la combinación con fórmula especial, hubo disminución de la producción de leche materna siendo necesario introducir una fórmula láctea de inicio para lactantes desde el segundo mes de edad, para aumentar el aporte de fenilalanina.

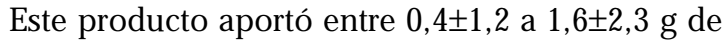
proteínas del día.

La recomendación de FA para lactantes menores de 6 meses de edad es 150 a $280 \mathrm{mg} /$ día (20-70 $\mathrm{mg} / \mathrm{kg} /$ día). En este estudio la ingesta promedio de FA fluctuó entre $119 \pm 25$ y los 290,4+59 mg/día (1 ${ }^{\mathrm{er}}$ y 60 mes de edad respectivamente). Al segundo mes de vida la ingesta promedio fue $186 \pm 57 \mathrm{mg} /$ día, de los cuales $154+66 \mathrm{mg} /$ día provenían de la leche materna y 23,4+59 mg/día de la fórmula láctea de inicio. Sin embargo, al sexto mes de edad, la FA estimada de la leche materna descendió a $107 \pm 114 \mathrm{mg} /$ día y el aporte de FA proveniente de la fórmula láctea de inicio se incrementó a $73 \pm 100$ $\mathrm{mg} /$ día. Al sexto mes se adiciona la FA proveniente de los alimentos sólidos permitidos (Tabla 3).

Es importante señalar que la FA proveniente de la fórmula de inicio adquiere importancia recién al quinto mes de edad, momento en que disminuye la producción de leche materna por baja estimulación de la mama (Figura 1). El volumen estimado de leche materna que ingieren en promedio al cuarto mes es $439 \pm 137 \mathrm{ml} /$ día, lo cual equivalía a $54 \%$ del volumen total de líquidos prescritos de acuerdo a las recomendaciones de la $\mathrm{RDA}$. Al sexto mes de edad, este volumen disminuyó a $348 \pm 275 \mathrm{ml} /$ día (14\% del volumen total), incrementándose el aporte de leche especial para PKU (Figura 2). Se pudo constatar que el uso de lactancia materna directa se mantuvo en $74 \%$ de la muestra a los 6 meses de edad.

En este estudio, 15 niños PKU (79\%) mantuvieron el nivel de FA en sangre entre 2 y $8 \mathrm{mg} / \mathrm{dl}$ durante los 6 meses de seguimiento. Cuatro casos (21\%) presentaron niveles de FA entre $10-12 \mathrm{mg} /$ $\mathrm{dl}$ en forma intermitente (Tabla 4).

En relación al estado nutricional, 84,2\% (16 de los niños PKU), mantuvo el P/E y T/E entre el puntaje $\mathrm{z}+1$ y -1 y $15,8 \%$ fue clasificado con sobrepeso durante los primeros seis meses de tratamiento. Hay que señalar que al segundo mes de seguimiento, 11\% (2 casos) presentó riesgo 


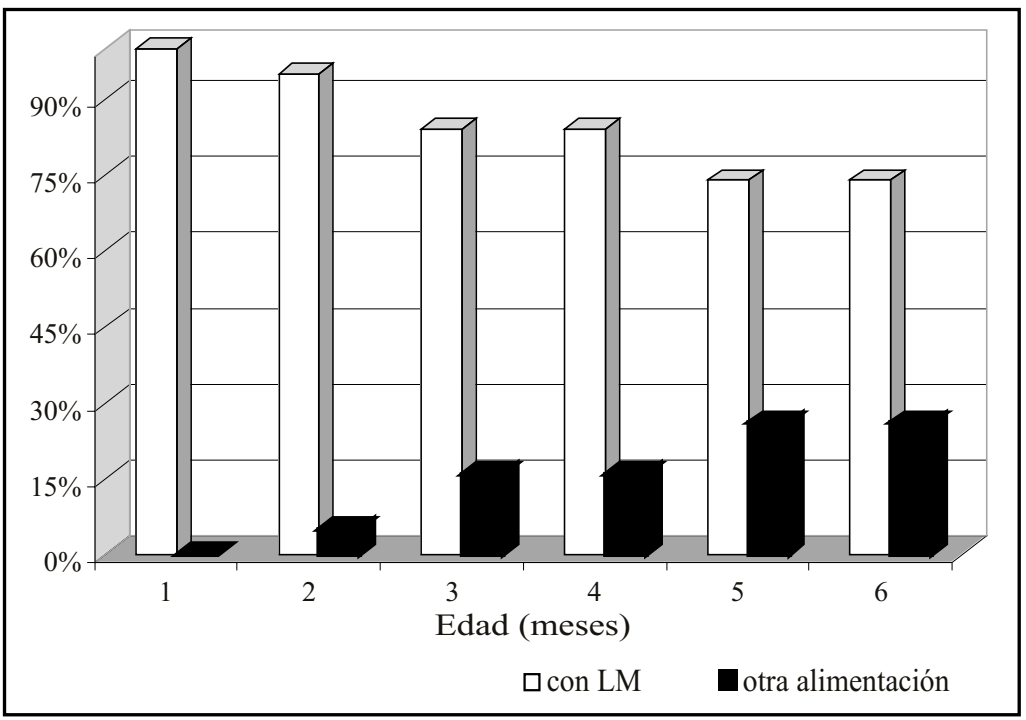

FiguRA 1. Porcentaje de prevalencia de lactancia materna (LM) en 19 niños PKU durante los 6 primeros meses de edad.

nutricional (Figura 3). Al evaluar P/T, a los 6 meses de edad, 63,2\% estaba eutrófico, 5,2\% estaba en riesgo nutricional y $31,8 \%$ tenía sobrepeso, sin embargo al sexto mes todos habían logrado un crecimiento normal (Figura 4).

Se evaluó el desarrollo psicomotor a los 4 meses en 10 niños PKU y a 6 PKU a los 6 meses de edad (escala de Bayley). El desarrollo mental (MDI) fue normal en $81 \%$ de los niños (13 niños). Cinco niños PKU tuvieron desarrollo motor bajo 80 puntos (Tabla 5).

\section{DiscUSIÓN}

Se ha demostrado ampliamente que el diagnóstico neonatal y la instauración precoz del tratamiento nutricional y la vigilancia estricta, permite que los niños PKU tengan crecimiento y desarrollo normales $^{1-3}$. Los resultados de este estudio demuestran que el manejo nutricional de los niños PKU de diagnóstico precoz, incorporando lactancia materna, permite un aporte adecuado de nutrientes, buen control metabólico, desarrollo pondoestatural y psicomotor normales.

La edad de diagnóstico de la muestra en estudio fue 19,29 $\pm 13,8$ días, considerado óptimo para evitar las secuelas neurológicas. Sin embargo, para prevenir el retardo mental a largo plazo, es necesario realizar el seguimiento médico, nutricional, bioquímico, neurológico y psicológico periódicos y permanentes ${ }^{15,16}$.

Estudios recientes han demostrado que el uso de la lactancia materna es una herramienta asequible para el adecuado manejo de niños con PKU consiguiéndose valiosos beneficios tales como

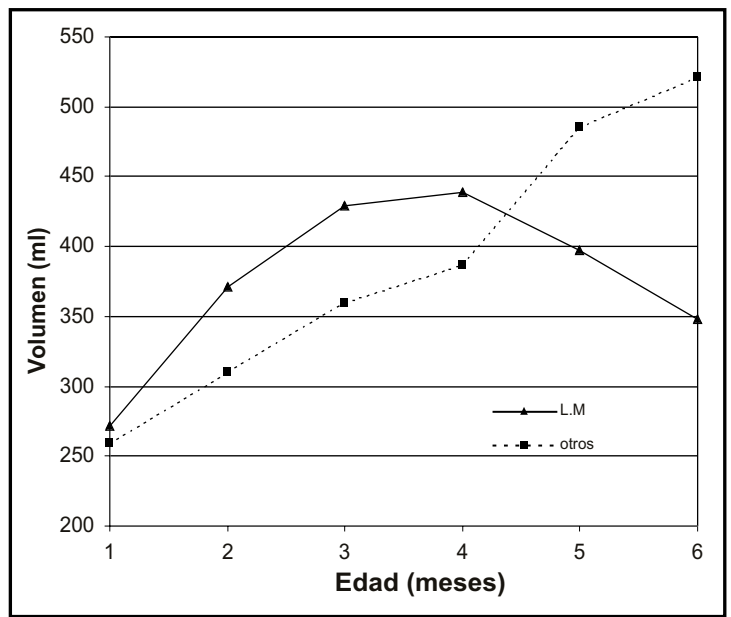

FigURA 2. Ingesta estimada de leche materna y otras fórmulas lácteas hasta los 6 meses de edad en niños PKU en seguimiento. 
Tabla 4. $\mathrm{N}$ iveles de FA en sangre en niños PKU durante los primeros 6 meses de edad

\begin{tabular}{|c|c|c|c|c|c|c|}
\hline $\begin{array}{l}\text { FA } \\
\text { sangre } \\
\text { (mg/dl) }\end{array}$ & $\begin{array}{c}1^{\text {er }} \text { mes } \\
n\end{array}$ & $\begin{array}{c}2^{\circ} \text { mes } \\
n\end{array}$ & $\begin{array}{c}3^{\text {er }} \text { mes } \\
n\end{array}$ & $\begin{array}{c}4^{\circ} \mathrm{mes} \\
\mathrm{n}\end{array}$ & $\begin{array}{c}\text { 5o mes } \\
n\end{array}$ & $\begin{array}{c}\text { 6o mes } \\
n\end{array}$ \\
\hline$<2$ & 4 & 3 & 3 & 5 & 3 & 3 \\
\hline 2 & 3 & 3 & 3 & 6 & 4 & 6 \\
\hline $2-4$ & 5 & 5 & 5 & 1 & 5 & 4 \\
\hline 4-6 & 2 & 5 & 1 & 2 & 4 & 3 \\
\hline $6-8$ & 1 & - & 3 & 1 & - & 2 \\
\hline $10-12$ & 4 & 2 & 3 & 4 & 3 & 1 \\
\hline Total (n) & 19 & 18 & 18 & 19 & 19 & 19 \\
\hline
\end{tabular}

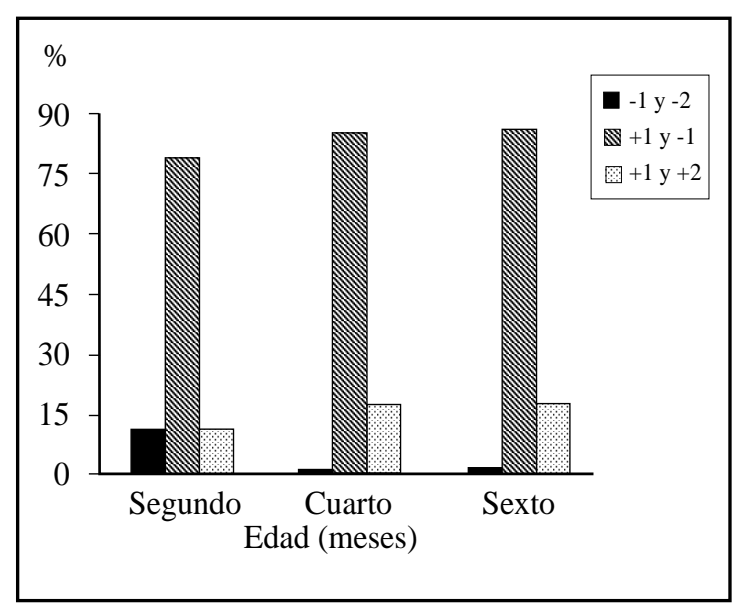

FiguRA 3. Peso para la edad (P/E) de 19 niños PKU de diagnóstico precoz (puntaje z).

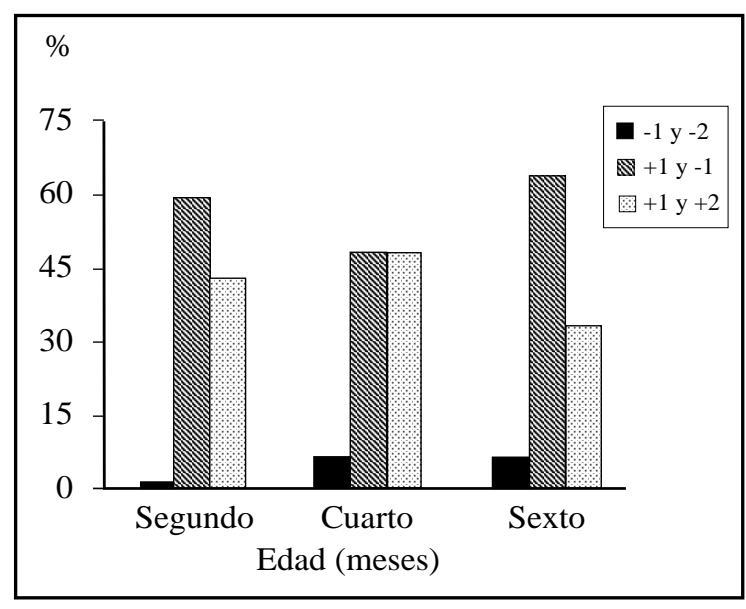

FiguRa 4. Peso para la talla en 19 niños PKU durante los 6 meses de seguimiento (puntaje $\mathrm{z}$ ).

Tabla 5. D esarrollo psicomotor de 16 niños PKU evaluados a los 4 y 6 meses de edad y en tratamiento con leche materna y fórmula sin FA. (Escala de Bayley)

\begin{tabular}{|lcrrrr|}
\hline $\begin{array}{l}\text { Edad } \\
\text { (meses) }\end{array}$ & \multicolumn{2}{c}{ MDI* $^{*}$} & \multicolumn{2}{c|}{ PDI $^{* *}$} & Total \\
\hline Cuarto & $<80$ & $>80$ & $<80$ & \\
Sexto & 2 & 8 & 8 & 2 & 10 \\
\hline
\end{tabular}

*Indice de desarrollo mental. **Indice de desarrollo motor. 
proporcionar menor cantidad de FA que las fórmulas lácteas infantiles y aportar nitrógeno no proteico para síntesis de proteínas, lo que favorece un mejor control metabólico de FA. Además, entrega ácidos grasos esenciales como el docosaexaenoíco (DHA), necesario para el desarrollo del cerebro y la visión ${ }^{8-10}$

Considerando que el tratamiento nutricional en la PKU no permite la ingesta de alimentos ricos en proteínas de alto valor biológico (carnes, huevo y aves, pescados, leche y derivados etc.), por su elevado contenido de FA, es necesario incorporar una fórmula especial sin o con menor cantidad de $\mathrm{FA}^{2}$. Al analizar la dieta de este grupo de pacientes PKU, se pudo constatar que la ingesta de proteínas durante los primeros 6 meses de edad cumplía las recomendaciones establecidas por la RDA para menores de 6 meses de edad (Tabla 2). La fórmula especial sin FA aportó aproximadamente $50 \%$ del total de las proteínas de alto valor biológico, mientras que la leche materna estimada el 35\%, lo cual demuestra la importancia del sustituto lácteo médico para el tratamiento de niños con PKU, ya que la disminución de leche materna necesaria para mantener el nivel de fenilalanina entre 2 y $6 \mathrm{mg} / \mathrm{dl}$, no cubre por si sola las necesidades de proteínas en este período de crecimiento.

$\mathrm{Al}$ analizar la ingesta dietaria de FA se pudo demostrar que durante el primer mes de tratamiento, 94\% de FA proviene de la leche materna y al sexto mes desciende a $37 \%$ del volumen total. La FA proveniente de las fórmulas de inicio adquieren importancia desde el cuarto mes de edad, cuando comienza a disminuir el volumen de leche materna de $54 \%$ a $14 \%$ del volumen total de líquidos requeridos, según edad y peso real del niño, al sexto mes de edad (Figura 2).

Otra información importante de destacar es que $74 \%$ (14 niños PKU) de la muestra mantuvo la lactancia materna directa hasta los 6 meses de edad, lo que corresponde al $24 \%$ de las proteínas diarias $(3,8 \pm 3,1 \mathrm{gr} /$ día $)$ y $36 \%$ del total de $\mathrm{FA} /$ día

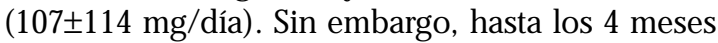

de edad el aporte de proteínas y FA de leche

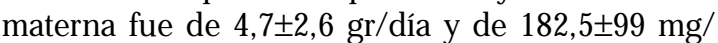
día respectivamente, lo que corresponde al 50\% de los requerimientos de ambos nutrientes. Esto nos permite concluir que la lactancia materna es importante en el tratamiento de los niños PKU hasta el $4^{\circ}$ mes de edad y que posteriormente, producto de la disminución de la producción de leche y al aumento de los requerimientos nutricionales de los niños, se hace necesario la incorporación de otros alimentos.

En relación al nivel en sangre de FA, 79\% de la muestra mantuvo excelente control metabólico. Se debe señalar que de los 4 niños que presentaron niveles elevados de FA y que correspondían a nivel socioeconómico y educacional bajo, sólo uno de ellos persistió con niveles sobre $10 \mathrm{mg} / \mathrm{dl}$ hasta los 6 meses de edad. Esto nos permite concluir que la educación y el seguimiento mensual que se realiza, ayuda a los padres a comprender, manejar y aplicar el tratamiento adecuadamente.

Greve y $\operatorname{cols}^{8}$, demostraron que el uso de leche materna permite crecimiento ponderal entre el puntaje $z+1$ y -1 . En este estudio, 63,2\% de los niños PKU era eutrófico, 31,6\% estaba con sobrepeso y $5,2 \%$ tenía riesgo nutricional a los 6 meses de edad, en tratamiento con leche materna y fórmula especial sin FA, según el indicador P/T.

Iniciar el tratamiento precozmente y mantener el nivel de FA en sangre bajo $10 \mathrm{mg} / \mathrm{dl}(600 \mu \mathrm{mol} /$ l) permite un desarrollo mental normal ${ }^{15-17}$. Los resultados de este estudio, demuestran que a los 4 y 6 meses de edad, 13 de los 16 pacientes evaluados (81\%) tenían MDI y PDI normal. Los niños PKU con PDI inferior a 80 correspondían a los de nivel socioeconómico y educacional bajo y con control metabólico fluctuante.

Se puede concluir que la lactancia materna para el tratamiento de niños con PKU de diagnóstico precoz, permite adecuado control metabólico, crecimiento ponderal en rangos de normalidad, lo cual favorece el óptimo desarrollo del niño y estimula la relación madre-hijo, mejorando asimismo la adherencia a la dieta y al tratamiento. 


\section{REFERENCIAS}

1. Scriver CH, Kaufmann S, Eisensmith R, Woo S. The hyperphenylalaninemias. En: Scriver Ch. Beaudet A. Sly W. Valle D, ed. The Metabolic and Molecular Bases of Inherited Disease, 7th. Edition. USA, editorial McGraw-Hill. Inc. Health Professions Division, 1995; 1015-77.

2. Cornejo V, Raimann E. Errores innatos del metabolismo de los aminoácidos. En: Errores innatos en el metabolismo del niño. Colombo M, Cornejo V, Raimann E, ed. Santiago: Editorial Universitaria, 1999; 59-66.

3. Colombo M, Troncoso L, Raimann E, Perales CG, Barros T, Cornejo V. Diagnóstico de fenilquetonuria en Chile. Rev Chil Pediatr 1988; 59: 235-9.

4. Guthrie R, Cornejo V. Programa de detección neonatal de errores innatos del metabolismo. En: Errores innatos en el metabolismo del niño, Editoras: Colombo M, Cornejo V, Raimann E. Santiago: Editorial Universitaria, 1999; 307-19.

5. Cornejo V, Raimann E, Brantes J, Lobo G, ABodowsky N, BecerRa C et al. The Chilean experience in newborn screening program for Phenylketonuria and Congenital Hypothyroidism. Infant Screening 1996; 19: 3-4.

6. Cornejo V, Becerra C, Brantes J, Lobo G, Pacheco J, Aвodovsky N ET AL. Normas para el óptimo desarrollo de programas de búsqueda masiva de PKU, Hipotiroidismo Congénito (HC) y otros errores congénitos del metabolismo. Santiago: Editorial Ministerio de Salud de Chile, 1999; 1-50.

7. Guthrie R, Susi A. A simple phenylalanine method for detecting phenylketonuria in large populations of newborn infants. Pediatrics 1963; 32: 334-8.
8. Greve L, Wheeler M, Green-Burgeson D, Zorn E. Breast-feeding in the management of the newborn with phenylketonuria: A practical approach to dietary therapy. J Am Diet Assoc 1994; 94: 305-9.

9. MotzFeld K, Lluje R, Nylander G. Breastfeeding in Phenylketonuria. Acta Paediatric Suppl 1999; 432: 25-7.

10. Riva E, Agostoni C, Biasucci G, Trjan S, Luotti D, FIORI L ET AL. Early breastfeeding is linked to higher intelligence quotient scores in dietary treated phenylketonuric children. Acta Paediatric 1996; 85: 56-8.

11. Agostoni C, Verduci E, Fiori L, Riva E, Giovannini M. Breastfeeding rates among hiperphenylalaninemic infants. Acta Pediatric 2000; 89: 366-7.

12. Acosta P, YanNicew S. Phenylketonuria (PKU) Protocol 1. En: Acosta P, Yannicelli S, ed. The Ross Metabolic Formula System. Nutrition Support Protocols, 4th Edition. Ohio, USA: Editorial Ross Laboratories 2001; 1-32.

13. National Research Council. Recommended Dietary Allowance 10th edition. Washington, DC: Editorial National Academy Press 1989; 247-61.

14. BARRERA G. Estándares Antropométricos para Evaluación del Estado Nutritivo. Santiago: Editorial Instituto de Nutrición y Tecnología de los Alimentos, Universidad de Chile, 2001; 1-119.

15. Smith I, Beasiey MG, Ades AE. Effect on intelligence of relaxing the low phenylalanine diet in phenylketonuria. Arch Dis Child 1991; 66: 311-6.

16. Koch R, Friedman E, Azen C. Report from the United States Collaborative Study for Children treated with Phenylketonuria. En: Bickel $\mathrm{H}$, Wachtel U, ed. Inherited Diseases of Aminoacid Metabolism. Springer Verlag, Stuttgart Alemania, 1985; 134-50.

17. BURGARD P. Development of intelligence in early treated phenylketonuria. Eur J Pediatr 2000; 159: S74-S79. 